\title{
Variabilidad en la implantación de las actividades comunitarias de promoción de la salud en España
}

\section{Variability in the implementation of health-promoting community activities in Spain}

\author{
S. March' ${ }^{1}$ M. Soler ${ }^{2}$, F. Miller ${ }^{3}$, I. Montaner ${ }^{4}$, M.J. Pérez Jarauta ${ }^{5,6}$, \\ M. $\operatorname{Ramos}^{7}$ (en nombre del grupo de investigación del PACAP)
}

\section{RESUMEN}

Fundamento. Describir la variabilidad en la realización de actividades comunitarias de promoción de la salud, en apoyos recibidos, en formación y en valoración de la participación de profesionales, administración y agentes sociales en las mismas.

Métodos. Estudio descriptivo mediante cuestionarios enviados a informadores clave identificados en cada CCAA con la ayuda de la Sociedad Española de Medicina de Familia y Comunitaria (SemFYC). El cuestionario incluía una definición de actividad comunitaria de promoción de la salud. Estudio realizado en 2008.

Resultados. Se observó una importante variabilidad en la realización de actividades comunitarias por CCAA. Los profesionales más implicados son las enfermeras y trabajadoras sociales. La mayoría de las CCAA (15 de 17) ofertan formación en estos temas a sus profesionales, sobre todo en educación para la salud. Es menos frecuente la formación a residentes de medicina de familia y comunitaria (10 de 17 CCAA) y en las diferentes especialidades de enfermería (6 de 17).

Los principales apoyos institucionales provienen de las gerencias de atención primaria, los servicios autonómicos de salud, los ayuntamientos, y los servicios de salud pública y los no institucionales de sociedades científicas, asociaciones ciudadanas y ONG. En la valoración de la implicación de diferentes entidades e instituciones, solo aprueban las ONG ( 3 sobre 5 ) y atención primaria (2,5 sobre 5).

Conclusiones. Existe unas alta variabilidad en las formas de apoyo e impulso a las actividades comunitarias en cada CCAA y consecuentemente en su difusión y puesta en práctica por los equipos de atención primaria. Enfermeras y trabajadoras sociales son agentes clave en este proceso.

Palabras clave. Promoción de la salud. Intervenciones comunitarias. Comunidad. Investigación en servicios de salud. Atención primaria.

\begin{abstract}
Background. To describe the variability in the application of community activities (CA) promoting health, in support received, in training, and in evaluation of the participation of professionals, administration and social partners in these.

Methods. Survey to key informants identified in each region with the help of the Spanish Society of Family and Community Medicine. The questionnaire included a definition of a health promotion community activity. The study was conducted in 2008 .

Results. There was variability in community activities by region. The most involved professionals are nurses and social workers. Most of the regions (15 of 17) offer training in these issues to their professionals, especially in health education. It is less frequent to offer training to family medicine residents (10 of 17 regions) and to the different nursing specialties (6 of 17).

The main institutional support comes from primary health care management, regional health services, municipalities, and public health services, and non-institutional support from scientific societies, civic associations and NGOs. On the evaluation of the involvement of different organizations and institutions in these ac tivities, the only entities receiving pass grades were NGOs (3 out of 5 ) and primary health care (2.5 out of 5).

Conclusions. There is a high variability in the ways of supporting and promoting community activities in each region and consequently in their dissemination and implementation by primary health care teams. Nurses and social workers are key players in this process.
\end{abstract}

Key words. Health promotion. Community activities. Community. Health service research. Primary health care.
1. Unidad de investigación GAP Mallorca, IB-Salut.

2. Centro de Salud Profesor Jesús Marín (Molina de Segura, Murcia).

3. Consultor Médico, Advance Medical, Barcelona.

4. Centro de Salud El Carmel, Barcelona, Institut Català de la Salut.

5. Instituto de Salud Pública y Laboral de Navarra

6. CIBER de Epidemiología y salud pública (CIBERESP).

7. Consejería de Salud y Consumo de las Illes Balears.

Recepción: 25 de junio de 2013

Aceptación provisional: 22 de julio de 2013

Aceptación definitiva: 29 de julio de 2013

\author{
Correspondencia: \\ Sebastià March Llull \\ Unidad de investigación GAP \\ C/ Reina Esclaramunda $n^{\circ} 9$ \\ 07003-Palma de Mallorca \\ Islas Baleares \\ E-mail: smarch@ibsalut.caib.es \\ Financiación \\ Este proyecto fue realizado por el Programa de Actividades Co- \\ munitarias en Atención Primaria (PACAP) de la Sociedad Española \\ de Medicina de Familia y Comunitaria (semFYC) y financiado por \\ la Agencia de Calidad del Sistema Nacional de Salud, del Ministerio \\ de Sanidad y Consumo (2008). \\ El estudio también ha contado con el apoyo del grupo de \\ investigación en servicios de la Red de investigación en activi- \\ dades preventivas y promoción de la salud de atención prima- \\ ria (RedIAPP, exp 06/0018/0036) y del grupo de investigación en \\ atención primaria del Instituto universitario de investigación en \\ ciencias de la salud de las Islas Baleares (IUNICS).
}




\section{INTRODUCCIÓN}

Las actividades comunitarias (AC) han demostrado ser eficaces para prevenir enfermedades y promocionar la salud ${ }^{1-4}$. La orientación comunitaria de los servicios de salud, recomendada por la $\mathrm{OMS}^{5,6}$, influyó en la reforma de la atención primaria (AP) española. Legislaciones de la última década han vuelto a subrayar la responsabilidad de AP en acción comunitaria ${ }^{7,8}$, aunque su implantación todavía no ha sido generalizada $^{9,10}$. En los últimos años, tanto en Espa$\tilde{n ̃ a}^{11,12}$ como en otros países ${ }^{13,14}$, ha resurgido el debate de promover las AC. Abordar este desafío exige conocer el punto de partida y señalar obstáculos y oportunidades.

Desde que en 2001 se finalizó en España la transferencia de competencias sanitarias a las comunidades autónomas (CCAA), cada sistema autonómico de salud puede desarrollar su propia política sanitaria, adaptando la organización a sus prioridades, fines y objetivos. Por lo tanto, es de esperar que la implantación de las $\mathrm{AC}$ en AP sea diferente entre CCAA.

Se han realizado algunos estudios sobre $\mathrm{AC}$ en los últimos años. En Cataluña, se describieron los 16 proyectos que formaban la red AUPA (Actuant units per la salud/ Actuando unidos por la salud) ${ }^{15}$. Gutiérrez y col ${ }^{16}$ describieron las actividades comunitarias y de educación para la salud realizadas por AP en una provincia del País Vasco. Aguiló y col analizaron la Red de Actividades Comunitarias (RAC) del Programa de Actividades Comunitarias en Atención Primaria (PACAP) de la SemFYC en 2002 cuantificando tipos de AC por $\mathrm{CCAA}^{17}$. En 2009, el estudio coordinado por Navarro y Martín Zurro sobre AP, recoge información sobre las estructuras de los servicios autonómicos que podrían facilitar las $\mathrm{AC}$ (p.e. capacidad de autogestión, implicación de la comunidad en la gestión de la AP) $)^{18}$.

Este trabajo se enmarca en una investigación realizada por el PACAP ${ }^{19}$ para describir la situación actual de las AC en España, y complementa a otro anterior ${ }^{20}$. Pretende describir la implantación de las AC por CCAA, su variabilidad, apoyos recibidos, formación y valoración de la participación de profesionales, administración y agentes sociales.

\section{MÉTODOS}

Estudio descriptivo mediante cuestionario a informantes clave en cada CCAA. La unidad de estudio es la CCAA. Los informantes fueron identificados por responsables de SemFYC/PACAP en cada CCAA. Su perfil era el de una o varias personas que por su trabajo y experiencia tuviesen una visión amplia y general sobre la implantación de AC en su CCAA. El cuestionario se remitió por correo electrónico y postal. Cada CCAA sólo devolvía un cuestionario aunque se animaba a diversificar las fuentes de información para completarlo. La recogida de información se realizó en 2008.

El cuestionario incluía una definición de AC20 y las siguientes variables: grado de participación de los equipos de atención primaria (EAP) en AC ( 5 niveles: menos del $20 \%$ de los EAP realizan AC, del 20 al 40\%; del 40 al $60 \%$, del 60 al $80 \%$ y más del $80 \%$ ); horario de realización (laboral, no laboral); implicación (puntuado del 0 al 5) de entidades cívicas (asociaciones de vecinos, ONG), colectivos profesionales y instituciones (gerencias, servicios autonómicos de salud, consejerías, salud pública); tipos de apoyos recibidos; existencia de consejos de salud activos (al menos 2 reuniones anuales) y estructuras de coordinación intersectorial; oferta de formación en AC; valoración de oportunidades y dificultades para realizar AC (puntuadas del 0 al 5), y propuestas de mejora (pregunta abierta).

Se realizó un análisis descriptivo y un análisis estratificado en función de si las CCAA tenían un nivel alto de participación (más del 60\% de los EAP colaboran en AC) o bajo (menos del $60 \%$ ).

\section{RESULTADOS}

Contestaron 29 informantes de las 17 CCAA, puesto que en tres de ellas (Aragón, Canarias y Madrid) contestaron un cuestionario entre varios informantes. En el resto sólo había un informante, aunque se desconoce si consultó otras fuentes para completar el cuestionario, tal y como se aconsejaba. Los perfiles de los informantes fueron 10 médicos de familia, 8 técnicos de 
educación para la salud, 5 enfermeras de $\mathrm{AP}, 2$ responsables de calidad y 3 de otros perfiles.

La variabilidad en la participación de los equipos de atención primaria (EAP) en $\mathrm{AC}$ se recoge en la figura 1 . Cuatro CCAA tienen un nivel alto de participación en AC (más del 60\% de los EAP). En la mayoría de las CCAA las AC se realizan tanto en horario laboral como fuera de éste. En Baleares, la Rioja y Madrid se realizan mayoritariamente en horario laboral, y en Andalucía fuera de este. Los profesionales más implicados en $\mathrm{AC}$ fueron las enfermeras y trabajadores sociales, aunque esta valoración variaba en función del grado de participación. El grado de implicación en AC valorado de 0-5 de las enfermeras era de 3,75 en las CCAA con alta partcipación en $\mathrm{AC}$, y de 2,67 en el resto, y para trabajadoras sociales de 3,75 y 1,83 respectivamente. Para el resto de profesionales también había diferencias: médicos 2 y 1,83 ; administrativos 1,5 y 0,17 ; y unidades de apoyo 2,25 y 0,92 .

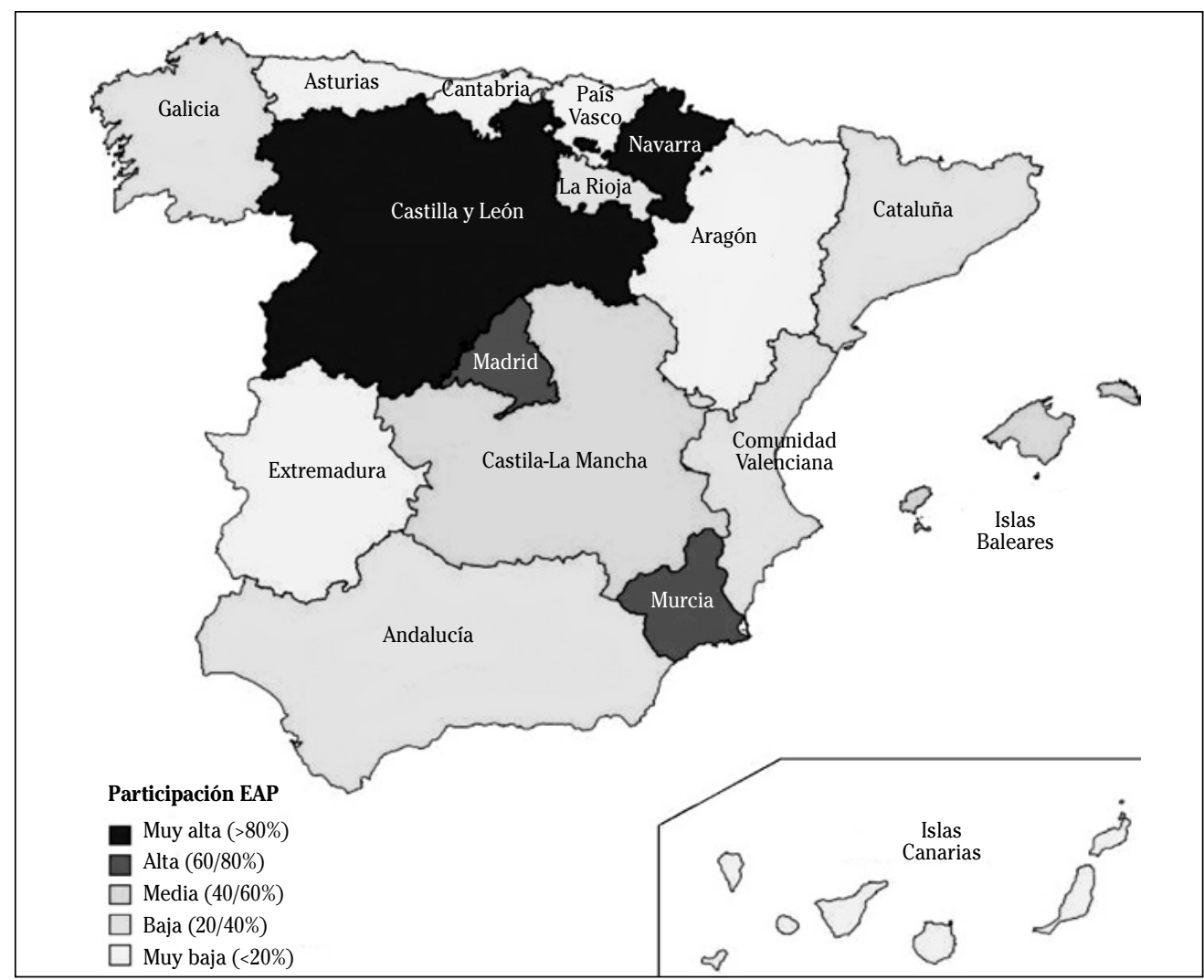

Figura1. Participación de los equipos de atención primaria en actividades comunitarias

\section{Apoyo a las actividades comunitarias}

En más de la mitad de las CCAA (10 de 17), todas o la mayoría de las gerencias de AP tienen entre sus objetivos formales alguno relacionado con promocionar las
AC. Solo la Comunidad Valenciana afirma no tenerlos en ninguna gerencia. La inclusión de estos objetivos es más frecuente en CCAA con participación alta en AC. Estos objetivos están en todas las consejerías autonómicas de salud excepto en las Islas 
Baleares. En 4 de ellas, además, se plantea específicamente la participación activa de los ciudadanos: a través de consejos de salud (Castilla León y Canarias), mediante procesos comunitarios (Extremadura) o sin especificar (País Vasco). Las AC están incluidas en cartera de servicios en Andalucía, Aragón, Cantabria, Islas Baleares, La Rioja, Madrid, Murcia, Navarra y País Vasco.

Todas las CCAA reciben algún apoyo institucional para desarrollar AC (Tabla 1), principalmente por parte de las gerencias de AP (14 CCAA), los servicios de salud autonómicos (13), los ayuntamientos (12) y los servicios de salud pública (11). Las CCAA con participación alta reciben apoyos de más instituciones. La inclusión de $\mathrm{AC}$ en la cartera de servicios, la formación de profesionales en $\mathrm{AC}$, y la elaboración y publicación de materiales, son más frecuentes en CCAA con participación alta en AC. En 13 CCAA, las AC reciben algún apoyo no institucional (Tabla 1), de sociedades científicas (11 CCAA), asociaciones ciudadanas (9), ONG (6), parroquia (5) y asociaciones profesionales (5). Las CCAA con participación alta en $\mathrm{AC}$ reciben más de estos apoyos.

Tabla 1. № de CCAA que reciben apoyos para realizar actividades comunitarias según entidad y tipo de apoyo*.

\begin{tabular}{lccccc}
\hline Apoyos institucionales & $\begin{array}{c}\text { Gerencias } \\
\text { de atención } \\
\text { primaria }\end{array}$ & $\begin{array}{c}\text { Salud } \\
\text { pública }\end{array}$ & $\begin{array}{c}\text { Sistemas } \\
\text { autonómicos } \\
\text { de salud }\end{array}$ & Municipio & TOTAL \\
\hline Formación profesionales & $11(65 \%)$ & $8(47 \%)$ & $10(59 \%)$ & $1(6 \%)$ & $17(100 \%)$ \\
\hline Cesión locales & $9(53 \%)$ & $3(18 \%)$ & $5(29 \%)$ & $8(47 \%)$ & $13(76 \%)$ \\
\hline Materiales usuarios & $4(23.5 \%)$ & $8(47 \%)$ & $5(29 \%)$ & $4(23.5 \%)$ & $10(59 \%)$ \\
\hline Materiales profesionales & $6(35 \%)$ & $9(53 \%)$ & $8(47 \%)$ & $3(18 \%)$ & $11(65 \%)$ \\
\hline Apoyo técnico & $10(59 \%)$ & $6(35 \%)$ & $5(29 \%)$ & $4(23.5 \%)$ & $13(76 \%)$ \\
\hline Premios/becas & $3(18 \%)$ & $3(18 \%)$ & $7(41 \%)$ & $2(12 \%)$ & $10(59 \%)$ \\
\hline Presupuesto específico & $2(12 \%)$ & $1(6 \%)$ & $4(23.5 \%)$ & $3(18 \%)$ & $9(53 \%)$ \\
\hline Liberar profesionales & $7(41 \%)$ & $3(18 \%)$ & $4(23.5 \%)$ & $2(12 \%)$ & $7(41 \%)$ \\
\hline Edición publicaciones & $5(29 \%)$ & $6(35 \%)$ & $7(41 \%)$ & $1(6 \%)$ & $10(59 \%)$ \\
\hline \multicolumn{1}{c}{ Total: } & $\mathbf{1 4 ( 8 2 \% )}$ & $\mathbf{1 1 ( 6 5 \% )}$ & $\mathbf{1 3 ( 7 6 \% )}$ & $\mathbf{1 2} \mathbf{( 7 1 \% )}$ & $\mathbf{1 7}(\mathbf{1 0 0 \% )}$ \\
\hline
\end{tabular}

\begin{tabular}{lccccc}
\hline $\begin{array}{l}\text { Apoyos no } \\
\text { institucionales }\end{array}$ & $\begin{array}{c}\text { Sociedades } \\
\text { científicas }\end{array}$ & ONG & $\begin{array}{c}\text { Asociaciones } \\
\text { ciudadanas }\end{array}$ & Parroquia & TOTAL \\
\hline Formación profesionales & $9(53 \%)$ & $3(18 \%)$ & $3(18 \%)$ & $1(6 \%)$ & $9(53 \%)$ \\
\hline Cesión locales & $5(29 \%)$ & $1(6 \%)$ & $8(47 \%)$ & $5(29 \%)$ & $8(47 \%)$ \\
\hline Materiales usuarios & $5(29 \%)$ & $5(29 \%)$ & $6(35 \%)$ & $2(12 \%)$ & $8(47 \%)$ \\
\hline Materiales profesionales & $7(41 \%)$ & - & $2(12 \%)$ & $1(6 \%)$ & $8(47 \%)$ \\
\hline Apoyo técnico & $2(12 \%)$ & $2(12 \%)$ & $3(18 \%)$ & $1(6 \%)$ & $3(18 \%)$ \\
\hline Premios/becas & $4(23.5 \%)$ & - & - & - & $4(23.5 \%)$ \\
\hline Presupuesto específico & $4(23.5 \%)$ & $4(23.5 \%)$ & $4(23.5 \%)$ & $1(6 \%)$ & $6(35 \%)$ \\
\hline Edición publicaciones & $6(35 \%)$ & $1(6 \%)$ & $2(12 \%)$ & - & $7(50 \%)$ \\
\hline \multicolumn{1}{c}{ Total: } & $\mathbf{1 1 ( 6 5 \% )}$ & $\mathbf{6 ( 3 5 \% )}$ & $\mathbf{9 ( 5 3 \% )}$ & $\mathbf{5 ( 2 9 \% )}$ & $\mathbf{1 1 ( 6 5 \% )}$ \\
\hline
\end{tabular}

* Los porcentajes se calculan sobre el total de CCAA (17). 
Doce CCAA tienen consejos de salud activos. Su número varía de uno en las Islas Baleares a 254 en Castilla León; Aragón y Canarias tenían 15, la Comunidad valenciana 23, Madrid 24, Navarra 31 y Extremadura 108. En las 4 CCAA restantes el número es desconocido. Trece CCAA declaran la existencia de mesas intersectoriales. Las instituciones que más participan son: asociaciones ciudadanas, AP, ayuntamientos y consejerías de salud (10 CCAA), seguidas de las consejerías de educación (9 CCAA). Participan más entidades en CCAA con alta participación en AC.

La implicación en las AC (valorado de 0 a 5) es media para asociaciones ciudadanas, ONG (puntuación mediana=3), y AP $(2,5)$, y baja para el resto: consejería de salud $(2,1)$, consejería de educación (2), ayuntamientos y servicios de salud $(1,5)$.

\section{Formación y difusión}

La mayoría de CCAA (15 de 17) oferta a profesionales de AP alguna actividad formativa relacionada con $\mathrm{AC}$. El contenido más frecuente es educación para la salud con grupos (13 CCAA), especialmente en CCAA con alta participación en AC. También se ofrecen cursos de iniciación (9
CCAA) y cursos avanzados de intervención comunitaria (8 CCAA), y formación en metodologías concretas como diagnóstico comunitario (4), AP orientada a la comunidad (4) e investigación acción participativa (4). Los residentes de medicina familiar y comunitaria reciben formación sobre $\mathrm{AC}$ en 11 CCAA y los residentes de enfermería o matronas en 6 CCAA.

En 10 comunidades se elaboraron materiales de apoyo a las AC dirigidos a profesionales y en 12 materiales para usuarios. Esto es más frecuente en CCAA con participación alta en AC. Para la difusión de las AC, los canales más utilizados son carteles y folletos (14 CCAA cada uno), y luego los medios de comunicación masiva (prensa 8 CCAA, radio 7 y TV 5) y las nuevas tecnologías (5).

\section{Valoración de las actividades comunitarias}

En opinión de los informantes, pesan más las dificultades (Fig. 2) que las oportunidades (Fig. 3) para realizar AC, especialmente la falta de tiempo, formación y motivación de los profesionales. Estas valoraciones son diferentes en función de si las CCAA tienen una participación en AC alta o baja.

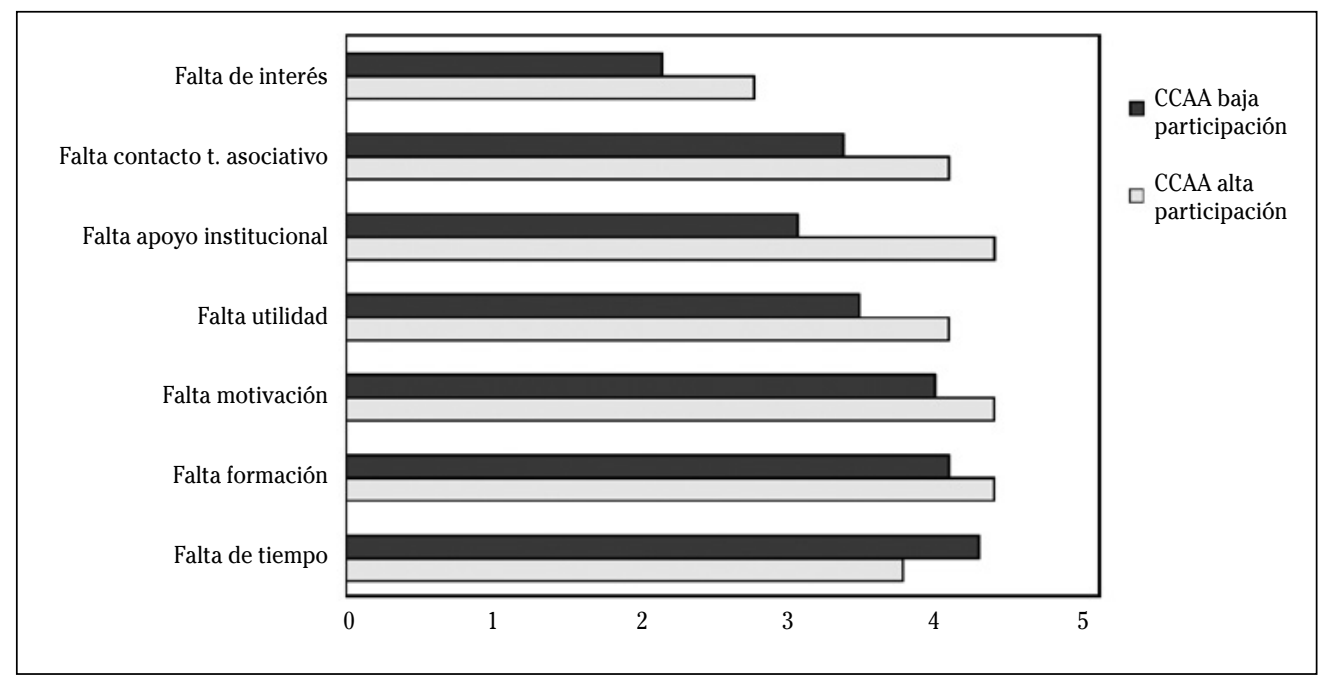

Figura 2. Dificultades para la realización de actividades comunitarias (de 0 a 5) 


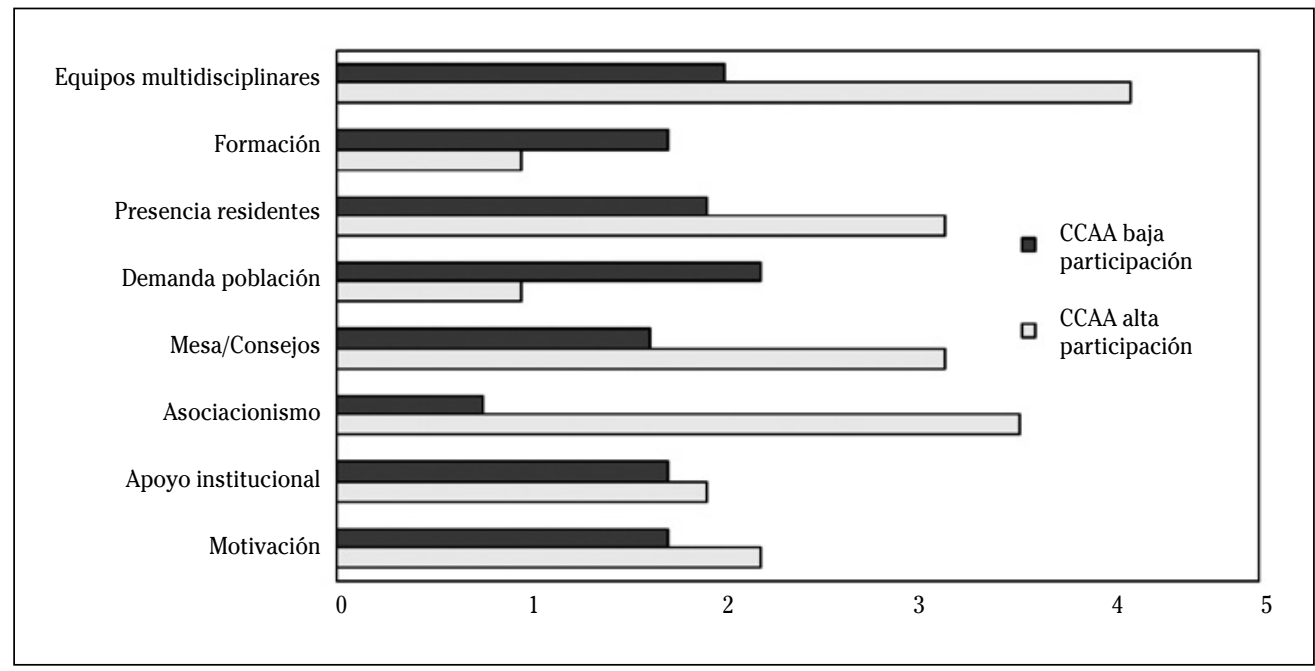

Figura 3. Oportunidades para la realización de actividades comunitarias (de 0 a 5 )

Por último, se recogieron propuestas sobre medidas para mejorar la situación actual de las AC. La mayoría de CCAA apuestan por mejorar la formación a nivel pregrado, postgrado y formación continuada. También se reclama mayor apoyo institucional, especialmente desde las consejerías de salud. Otras propuestas se refieren a incluir las AC en cartera de servicios, mejorar la coordinación intersectorial, potenciar las redes de AC, aumentar la difusión y visibilidad de $\mathrm{AC}$, e incentivar los proyectos y a los profesionales que las realizan.

\section{DISCUSIÓN}

El principal resultado del estudio ha sido constatar la alta variabilidad autonómica en la implantación de AC. Se confirma el liderazgo de enfermería en el desarrollo de estas actividades y se recoge que los agentes implicados más valorados son las ONG y las gerencias de AP. El estudio describe el alcance de los apoyos que reciben las AC y la formación que reciben los profesionales en cada CCAA, y subraya las diferencias en la valoración de dificultades y oportunidades para su realización, dependiendo de si son CCAA que realizan más o menos AC.
El estudio se realizó mediante cuestionarios a personas expertas. Esta metodología ha sido utilizada en estudios similares de valoración de servicios sanitarios ${ }^{18}$ demostrando ser muy efectiva para alcanzar una visión general de la variabilidad autonómica de prácticas, aunque los resultados reflejan opiniones, lo cual supone una limitación. Las CCAA de mayor tamaño podrían tener una importante variabilidad interna respecto a las AC. En el ánimo de evitarlo, se invitó a los informantes a contactar con otros profesionales para completar el cuestionario. En algunas CCAA, como Madrid, el informador consultó con personas clave en cada área de salud, para después completar un solo cuestionario sintetizando opiniones. En otras, como Andalucía, contestó una sola persona sin que haya constancia de que consultase con nadie más, por lo que la información podría ser menos fiable. A pesar de estas limitaciones, participaron informantes clave de todas las CCAA excepto de las ciudades autónomas de Ceuta y Melilla con las que no se logró contactar.

Los resultados evidencian la variabilidad existente entre CCAA respecto a la implantación de AC. Esto lo explican las diferentes prioridades que las administraciones sanitarias autonómicas han otorgado 
a la promoción de la salud y a la participación comunitaria. El interés y apoyo de estas administraciones ha sido destacado como un factor clave para la realización de $\mathrm{AC}^{15,21}$. Muchas CCAA todavía no tienen objetivos formales en sus gerencias de atención primaria que contemplen la realización de AC, ni incluyen estas acciones en las carteras de servicios de sus centros de salud, ni tienen mesas intersectoriales institucionalizadas, aunque este último es un elemento fundamental para el desarrollo de $\mathrm{AC}^{22,23}$. Cabe recordar que la acción comunitaria y el trabajo intersectorial para la promoción de la salud son responsabilidades de la AP según la legislación vigente ${ }^{8}$.

Cuatro CCAA tienen un nivel alto de participación en AC (más del $60 \%$ de los EAP las realizan). Estas tienen algunas características que las diferencian de las demás. En primer lugar, todas incluyen entre los objetivos de sus gerencias la promoción de $\mathrm{AC}$, las cuales están incluidas en cartera de servicios de los centros de salud. En segundo lugar, disponen de más apoyos institucionales para la formación de profesionales y la elaboración y publicación de materiales, junto con mayor número de apoyos no institucionales en general. En tercer lugar, sus mesas intersectoriales son más plurales, participa mayor variedad de entidades. Estas características coinciden con las identificadas por otro estudio realizado en equipos que realizaban $\mathrm{AC}$ en Cataluña $a^{15}$. Los resultados sugieren que un apoyo decidido por parte de gerencias de AP y administración sanitaria autonómica ayudaría a impulsar la realización de AC. Es necesaria más investigación para conocer los factores que influyen en la realización de las AC.

Los resultados confirman el liderazgo de enfermería en el desarrollo de las AC en AP, ya identificado en otros estudios ${ }^{15,20}$. Además, se resalta la importancia de las trabajadoras sociales en aquellas CCAA que realizan más intervenciones comunitarias. Esta parece variar en función del rol que tienen las trabajadoras sociales en cada CCAA. Mientras que en algunas, como Navarra o Castilla la Mancha, las trabajadoras sociales son fundamentales para de- sarrollar estas actividades, en otras, como Baleares, sus funciones están más orientadas hacia tareas burocráticas, sin demasiado impacto en las AC. Esto sugiere que una determinada definición del rol que desempeñan junto con una correcta integración en el equipo del centro de salud y la desburocratización de sus tareas, podría ser una estrategia para desarrollar AC.

La mayoría de las CCAA ofrecen formación específica sobre intervenciones de promoción de la salud aunque apenas hay formación en pregrado. La falta de formación es una de las dificultades más priorizadas, tanto en este estudio como en otros $^{24,25}$. Aunque no hay muchas CCAA que elaboren materiales de promoción de la salud, hay que destacar la oportunidad que supone que algunas, como Navarra ${ }^{26}$ o Madrid, que poseen tienen una amplia experiencia y que permiten que sus materiales puedan ser usados por otras.

Aunque las gerencias de atención primaria y los servicios autonómicos de salud son los agentes institucionales que brindan apoyos en más CCAA, sólo las primeras obtienen un aprobado en su valoración, mientras que servicios de salud, consejerías y ayuntamientos suspenden. Destaca lo que parece una baja implicación de los servicios de salud pública autonómicos en la promoción de AC cuando podría ser una interesante línea de intervención conjunta con AP. Esta parece ser la apuesta de algunas sociedades científicas como la SemFYC y la Sociedad Española de Salud Pública y Administración Sanitaria (SESPAS) ${ }^{27-28}$. Este aspecto debería revisarse en las reformas de salud pública que actualmente se están desarrollando ${ }^{29}$. Es interesante impulsar estas alianzas estratégicas en el actual contexto de crisis económica, puesto que las acciones de promoción de la salud desarrolladas de esta manera pueden resultar altamente eficientes a largo plazo tal y como advierte la $\mathrm{OMS}^{30}$.

Las barreras y oportunidades para el desarrollo de las AC identificadas, son similares a las obtenidas en otros estudios con profesionales ${ }^{15,23}$. Llaman la atención las diferentes valoraciones que hacen las CCAA con alto nivel de participación en AC 
(más del 60\% de los EAP) y las que tienen un nivel más bajo (menos del 60\%). Mientras la demanda ciudadana fue la oportunidad más destacada para CCAA con bajo nivel de participación, en las de alta participación destacaron la multidisciplinariedad del equipo y el asociacionismo de la población (Fig. 4). Para las CCAA con baja participación en AC la principal dificultad fue la falta de tiempo, mientras que para las de alta, era la falta de apoyos (Fig. 3).

Los resultados del estudio advierten de una importante desigualdad en la oferta de servicios de promoción de la salud en el ámbito comunitario en la AP española. Además, apuntan a que mejorar apoyos de las instituciones implicadas y el asociacionismo de la misma comunidad podrían ayudar a desarrollar las AC. Se confirma el liderazgo de enfermería en $\mathrm{AC}$, identificando al trabajador social como otro agente clave, con un rol comunitario que varía mucho entre CCAA.

\section{Agradecimientos}

Los autores agradecen la colaboración de Rafa Cofiño y del blog saludcomunitaria.wordpress.com, la de Xavier Moyà, y la de los informantes clave participantes en el estudio. Asimismo, agradecen el apoyo facilitado por Semfyc.

\section{BIBLIOGRAFÍA}

1. Agencia de Evaluación de Tecnologías Sanitarias (AETS), Instituto de Salud Carlos III. Ministerio de Sanidad y Consumo. Evaluación de la efectividad de tecnologías para la promoción de la salud y prevención de la enfermedad. Madrid: AETS-Instituto de Salud Carlos III. 2002.

2. Cofiño R, Álvarez B, Fernández S, Hernádez R. Promoción de la salud basada en la evidencia: ¿realmente funcionan los programas de salud comunitarios? Aten Primaria 2005; 35 : 478-483.

3. The Community Guide [internet]. USA government; [actualizado en 10/5/2012; consultado en 4/6/2012]. Disponible en: http://www. thecommunityguide.org/

4. International Union for Health Promotion and Education (IUHPE). La evidencia de la eficacia de la promoción de la salud. Configurando la Salud pública en una nueva Europa. IUHPE. Traducido por Subdirección General de Promoción de la Salud y Epidemiología, de la Dirección General de Salud Pública Consumo y del Ministerio de Sanidad y Consumo, España. Disponible en: http://www. msc.es/profesionales/saludPublica/prevPromocion/docs/Parte_1.pdf

5. Organización Mundial de la salud. Declaración de Alma Ata. OMS; 1978. [consultado 19/5/2012]. Disponible en: http://www.paho. org/Spanish/dd/pin/alma-ata_declaracion.htm

6. Organización Mundial de la Salud. Carta de Bangkok. OMS; 2005. Disponible en: www.who. int/healthpromotion/conferences/6gchp/ BCHP_es.pd.

7. Ley $16 / 2003$ de 28 de Mayo de Cohesión y Calidad del Sistema Nacional de Salud. Boletín Oficial del Estado, nº 128 (29-5-2003).

8. Real Decreto $1030 / 2006$ del 15 de septiembre por el que se establece la cartera de servicios comunes del Sistema Nacional de Salud y el procedimiento para sua ctualización. Boletín Oficial del Estado, nº 222 (16-9-2006).

9. Gervás J, Pérez-Fernández M, Palomo-Cobos L, PASTOR R. Veinte años de reforma de la atención primaria en España. Valoración para un aprendizaje por acierto / error. Madrid: Ministerio Sanidad y Consumo 2005; Disponible en: www.msc.es.

10. Soler Torroja M. Promoción de la salud, atención comunitaria y medicina de familia. Aten Primaria 2005; 36: 355-357.

11. IRIgoYen J. Perspectivas de la participación en salud después de la reforma gerencialista. Comunidad 2004; 7: 73-79.

12. Turabián JL, Pérez B. Grandes misterios. ¿Puedes ver al monstruo del lago Ness?: el modelo biopsicosocial y las actividades comunitarias. Aten Primaria 2007; 39: 261-264.

13. NICE. Community engagement to improve health. London, Nice public health guidance 9, 2008. [consultado 11 de marzo de 2012]. Diponible en: http://www.nice.org.uk/nicemedia/pdf/PH009Guidance.pdf.

14. Contryhealthrankings.org [internet] Wisconsin: University of wisconsin Population Health Institute. [consultado 11 de marzo de 2012]. Disponible en: www.countyhealthrankings.org/take-action\%20.

15. López E, Forcada C, Miller F, Pasarín Rua Mi, Foz GIL G. Factores que facilitan y dificultan el desarrollo de los proyectos comunitarios. Estudio observacional de la red AUPA de centros de atención primaria de Catalunya. Aten Primaria 2010; 42: 218-225. 
16. Gutierrez M, Azpeitia ME, Vergara I. Actuar con la comunidad... ¿Una asignatura pendiente en Osakidetza? Comunidad 2004; 7: 63-67.

17. Aguiló Pastrana E, López Martín M, Siles Román D, LóPEz FERNÁDEZ LA. Las actividades comunitarias en atención primaria en España. Un análisis a partir de la Red del Programa de Actividades Comunitarias (PACAP). Aten Primaria 2002; 29: 26-32.

18. Regato P, Dominguez J, Sancho M. Coordinación sanitaria y abordaje comunitario en atención primaria. En: Navarro V, Martin-Zurro A, coordinadores. La atención primaria de salud en España y en sus comunidades autonómicas. Barcelona: Semfyc 2009; 149-168.

19. March S, Bauż̀ LL, Ruiz-Jiménez JL, Soler M, MonserRAT MR. Consideraciones sobre el informe de la situación de las actividades comunitarias en atención primaria. Comunidad 2010; 12: 6-10.

20. March S, Ramos M, Soler M, Ruiz-Jiménez JL, MiLLER F, DOMÍNguez J y grupo de investigación PACAP. Revisión documental de actividades comunitarias realizadas en atención primaria. Aten Primaria 2011; 43: 289-296.

21. GULDAN GS. Obstacles to community health promotion. Soc Sci Med 1996; 43: 689-695.

22. Mercel C, D’Afflitti J. Reconsiderating community-based health promotion: promise, performance and potential. Am J Public Health 2003; 93: 557-574.

23. NutBEAm D. Inter-sectorial action for health: making it work. Health promot int 1994; 9: 143-144.
24. DiAZ G. Factores relacionados con la práctica de educación sanitaria por los médicos de atención primaria de Galicia. Rev Esp Salud Publica 2001; 75: 529-540.

25. Myrand A, Beehler G, Kuo C, Mahoney M. Explaining the de-prioritization of primary prevention: physicians' perceptions of their role in the delivery of primary care. BMC Public Health 2003; 3: 5 .

26. Gobierno de Navarra [sede web]. Pamplona: Gobierno de Navarra. 2012 [accedido en 10/7/2011]. Publicaciones de promoción de la salud. http://www.navarra.es/ home_es/Gobierno+de+Navarra/Organigrama/Los+departamentos/Salud/Organigrama/Estructura+Organica/Instituto $+\mathrm{Na}$ varro+de+Salud +Publica/Publicaciones/ Planes+estrategicos/Promocion+de+la+Salud/

27. Sociedad española de medicina de Família y Comunitaria. Barcelona, Semfyc. 2012. Noticias [accedido en 10/7/2011]. http://www. semfyc.es/es/noticias/destacadas/listado/ SESPAS_IConferenciaSaludComunitaria/

28. Cofiño R, Pasarín MI, Segura A. ¿Cómo abordar la dimensión colectiva de la salud de las personas? Informe SESPAS 2012. Gac Sanit 2012: 26 (Supl): 88-93.

29. Hernández-Aguado I, Benavides FG, Porta M. Los profesionales españoles de la Salud Pública ante la Ley General de Salud Pública. Gac sanit 2012; 26: 295-297.

30. OMS. Informe sobre la salud en el mundo 2010. La financiación de los sistemas de salud. Suiza: OMS; 2010. 
